\title{
Importance of EDTA in the Detection of Metallo Beta Lactamase from Imipenem Resistant Gram Negative Bacilli
}

\author{
P. Kanchanadevi ${ }^{1 *}$ and S. Chandra Sekaran ${ }^{2}$ \\ ${ }^{1}$ CSI College of Dental Sciences and Research, Eastveli Street, Madurai, India \\ ${ }^{2}$ NIMRA Institute of Medical Sciences, Jupidi village, Ibrahimpatnam, Mandal, \\ Krishna DT, Vijaywada, Andhrapradesh, India \\ *Corresponding author
}

\begin{tabular}{|c|c|}
\hline & A B S T R A C T \\
\hline Keywords & \multirow{4}{*}{$\begin{array}{l}\text { Gram negative Bacilli isolates resistant to imipenem were screened } \\
\text { phenotypically for Metallo Beta Lactamase( MBL) production by EDTA } \\
\text { Disc Synergy (EDS) method and MIC reduction test. EDTA, a chelating } \\
\text { agent which inhibits the production of MBL and hence incorporation of } \\
\text { EDTA to the resistant strains, results in the zone formation. In this study, } \\
\text { Minimal Inhibitory Concentration for Imipenem was also estimated and the } \\
\text { incorporation of EDTA to the drug dilution plates differentiated all MBL } \\
\text { producing Gram negative Bacilli. }\end{array}$} \\
\hline $\begin{array}{l}\text { MBL(Metallo Beta } \\
\text { Lactamase),MIC } \\
\text { EDTA (Ethylene } \\
\text { DiamineTetra } \\
\text { Aceticacid). }\end{array}$ & \\
\hline Article Info & \\
\hline $\begin{array}{l}\text { Accepted: } \\
\text { 26 October } 2016 \\
\text { Available Online: } \\
\text { 10 November } 2016\end{array}$ & \\
\hline
\end{tabular}

\section{Introduction}

EDTA (Ethylene Diamine Tetra Acetic acid) is a colorless water soluble solid. It is a chelating agent. Imipenem resistance mediated by acquired MBLs has been reported in many countries. MBL producing Imipenem resistant strains may spread rapidly to other species of gram negative bacilli. Since EDTA, inhibits the production of MBL, the incorporation of EDTA to the Imipenem resistant strains resulted in susceptibility.

Minimal Inhibitory Concentration is the least concentration of an antimicrobial agent which inhibits the growth of a microorganism after overnight incubation.
The use of EDTA along with antibiotics in MIC reduction test is useful for the detection of MBL production.

EDS test using Imipenem and EDTA disc have been reported as a simple method to detect MBL producing clinical isolates. The aim of this study is to determine the use of EDTA in the detection of MBL from imipenem resistant isolates of Gram negative Bacilli.

\section{Materials and Methods}

This study was conducted over a period of 3 years (Jan 2013 - Jan 2016) at Christian 
Mission Hospital, Madurai. A total of 76 E.coli sp., 60 Pseudomonas sp., 9 Klebsiella sp., 3 Proteus sp., 1 Citrobacter sp., 2 Salmonella typhi sp., and 1 Shigella sp., These organisms were isolated from various samples like Urine, Pus, Blood, Stool and Sputum of both outpatients and inpatients admitted to different wards, were sent to the laboratory for diagnosis and sensitivity testing.

All these isolates were undergone antibiogram for imipenem resistance by the routine Kirby- Bauer Disc diffusion method using CLSI manual. A Total of 25 E.coli, 42 Pseudomonas, 2 Klebsiella,1 Proteus and 1 Citrobacter were resistant to Imipenem. Their MIC values were also found. The imipenem resistant strains were subjected to EDS test and MIC reduction test using EDTA.

\section{EDTA disk synergy (EDS) test}

EDTA disk synergy (EDS) test was done with imipenem and EDTA discs for detection of Metallo Beta Lactamases in the imipenem resistant isolates.

A $0.5 \mathrm{M}$ EDTA solution was made by dissolving 1.86.g of disodium EDTA. $2 \mathrm{H}_{2} \mathrm{O}$ (NICE CHEMICALS, Kerala India )in 10 $\mathrm{ml}$ of distilled water. The $\mathrm{pH}$ was adjusted to 8.0 by adding either $\mathrm{Hcl}$ or $\mathrm{NaOH}$ and should be sterilised by autoclave.

The test isolate of 18-24 hours culture was adjusted to $0.5 \mathrm{McFarland}$ standard and spread on the surface of a MHA plate. A 10ug imipenem disc (HI-MEDIA, Mumbai, India) was placed on the agar. A blank disk prepared from Whatmann filter paper no. 1 had incorporated with 10ul of $0.5 \mathrm{M}$ EDTA was kept $10 \mathrm{~mm}$ edge-to-edge apart from the imipenem disc. After incubating overnight at $37^{\circ} \mathrm{C}$, the appreance of zone between the two discs was interpreted as positive for MBL production.

\section{MIC Reduction test}

MIC reduction test of imipenem was done by agar dilution method. EDTA $(1 \mathrm{ml}$ solution of $0.5 \mathrm{M}$ ) was added to $1 \mathrm{ml}$ of the imipenem. Mix $2 \mathrm{ml}$ of EDTA and imipenem with $18 \mathrm{ml}$ of molten Mueller Hinton agar and poured on plates that were allowed to set. A loopful of test inoculums was spot inoculated on these plates. The reading was taken after overnight incubation. The highest dilution of imipenem that inhibits the growth of the organism was taken as MIC. The four fold reduction from the previous MIC of these strains without EDTA confirmed that the strains were MBL producer.

\section{Results and Discussion}

A total of 154 isolates of 7 various gram negative Bacilli (76 E.coli sp., 60 Pseudomonas sp., 9 Klebsiella sp., 3 Proteus sp., 1 Citrobacter sp., 2 Salmonella typhi and1 Shigella sp.,) were included in the study. A total of 25 E. coli, 42 Pseudomonas, 2 Klebsiella, 1 Proteus and 1 Citrobacter were found to be Imipenem resistant and the rest of the isolates showed sensitivity by the routine antibiogram. Minimal inhibitory concentration of these strains for Imipenem is shown in Table 1.

All the 71 imipenem resistant strains ( 25 E.coli,42 Pseudomonas sp., 2 Klebsiella sp., 1 Proteus sp., 1 Citrobacter sp., ) were screened for MBL production by EDS test and MIC reduction test. Among the 25 imipenem resistant E.coli sp., 12 (48\%) were found to produce MBL by EDS test and $15(60 \%)$ were found to produce MBL by MIC reduction test. Among the 42imipenem resistant Pseudomonas sp., 19(45.23\%) were found to produce MBL by EDS test and 23(54.76\%) were found to produce MBL by MIC reduction test. 
Antibiotic resistance may be due to the mechanisms like lack of drug penetration, mutation, efflux mechanism and the production of enzymes. Of which the production of MBL is of great importance in imipenem resistance among gram negative bacilli.
MBL producing gram negative bacilli may transfer resistance gene to other microorganisms leading to serious medical problems in hospitals, all over the world. Hence the detection MBL producing gram negative bacilli at the very early period is important to prevent dissemination.

Table.1

MIC For Imipenem

\begin{tabular}{|c|c|c|c|c|c|c|c|c|c|c|c|c|c|}
\hline & $>256 \mathrm{mg} / \mathrm{l}$ & $256 \mathrm{mg} / \mathrm{l}$ & $128 \mathrm{mg} / \mathrm{l}$ & $64 \mathrm{mg} / \mathrm{l}$ & $32 \mathrm{mg} / \mathrm{l}$ & $16 \mathrm{mg} / \mathrm{l}$ & $8 \mathrm{mg} / \mathrm{l}$ & $4 \mathrm{mg} / \mathrm{l}$ & $2 \mathrm{mg} / \mathrm{l}$ & $1 \mathrm{mg} / \mathrm{l}$ & $0.5 \mathrm{mg} / \mathrm{l}$ & $0.25 \mathrm{mg} / \mathrm{l}$ & $0.125 \mathrm{mg} / \mathrm{l}$ \\
\hline E.coli (25) & 1 & 1 & 6 & 8 & 5 & 1 & & & 1 & & 1 & 1 & \\
\hline Pseudomonas (42) & 8 & 4 & 13 & 4 & 5 & 1 & 1 & 1 & 1 & & 1 & 2 & 1 \\
\hline Proteus (1) & & & & & & & & & 1 & & & & \\
\hline Klebsiella (2) & & & & 2 & & & & & & & & & \\
\hline & & & & & & & & & & & & & \\
\hline Citrobacter (1) & & & & 1 & & & & & & & & & \\
\hline
\end{tabular}

EDTA, Ethylene Diamine Tetraacetic Acid, is used in many ways like for chelation treatment, lead poisoning, multiple sclerosis, Parkinson's disease. Scleroderma, Psoriasis, Eye infection etc.. It is also used to improve stability in pharmaceutical products. It is also used in certain blood collection tubes in laboratories (WebMD). EDTA also inhibits the production of MBL and hence it is used in this study.

In EDTA Disc synergy test, EDTA inhibits MBL from Imipenem resistant strain and hence there was zone formation. $48 \%$ of E.coli and $45.23 \%$ of Pseudomonas were found to produce MBL by this method. In the study of Envuru et al., (2011), 50\% of MBL production is given by E.coli. Similar results of $46 \%$ of $\mathrm{MBL}$ production in Pseudomonas could be detected in the study of Johann Pitout et al., (2005). Noyal et al., (2009) had reported 50\% of MBL production in Pseudomonas. In contrast, MBL production in Pseudomonas in the study of Varaiya et al., (2008), Agarwal et al., (2008), Navneeth et al., (2002), Hemalatha et al., (2005), Mandiratta et al., (2005) were 20.8\%, $8.05 \%$,
$12 \%, 14 \%, 8.62 \%$ respectively.

E.coli (76\%) showed their MIC for Imipenem ranges from $128 \mathrm{mg} / \mathrm{l}$ to $32 \mathrm{mg} / \mathrm{l}$ and Pseudomonas (81\%) showed their MIC for Imipenem ranges from $>256 \mathrm{mg} / 1$ to $32 \mathrm{mg} / \mathrm{l}$. Addition of EDTA to MIC dilution plates showed the difference in ranges and there was four fold reduction in the results and thus confirmed the production of MBL. 2 Klebsiella sp., 1 Proteus sp.,1 Citrobacter sp., used in this study showed imipenem resistance by Kirby Bauer disc diffusion method but produced negative result for MBL production.

Though EDTA is used in various ways, this study reveals the usage of EDTA in the detection of MBL production from imipenem resistant Gram negative Bacilli. The inclusion of MBL detection test in the routine diagnosis may eliminate the dissemination of antibiotic resistance.

\section{References}

Agarwal, G., R.B. Lodhi, et al., Study of Metallo Beta Lactamase Production in 
Clinical Isolates of Pseudomonas aeroginosa, Indian J. Med. Microbiol., 26(4)P: 349-51.

Beth A. Rasmussen and Karen Bush, Carbapenem Hydrolysing Beta Lactamases, Antimicrobial Agents and Chemotherapy, Feb.1997,p 223-232.

Clinical and Laboratory Standard Institute. 2006. Methods for Dilution Antimicrobial Susceptibility Tests for Bacteria that Grow Aerobically; Approved Standard - Seventh Edition. M7- A7,Vol 26, No.2.

Dongeun Yong, Kyungwon Lee et al., 2002. Imipenem EDTA Disk Method for Differentiation of Metallo Beta Lactamase Producing Clinical Isolates of Pseudomonas sp., and Acinetobacter sp., J. Clin. Microbiol., 3798-3801.

Enwuru, N.V., Enwuru, C.A., Ogbonnia, S.O., Adepoju-Bello, A.A. 2011. Metallo-B-Lactamase Production by Escherichia Coli and Klebsiella Species Isolated from Hospital and Community Subjects in Lagos, Nigeria. Nature and Sci., 9(11).

Hae Suk Cheong, Cheol in kang et al., 2007. Clinical significance of healthcare associated infections in community onset Escherichia coli bacteraemia. J. Antimicrobial Chemotherapy, 60: 1355-1360.

Hemalatha, V., Sekar, U., Kamat, V. 2005. Detection of metallo beta latamase producing Pseudomonas aeroginosa in hospitalised patients. Indian J. Med. Res., 122: 148-52.

Hisakazu Yano, Akio Kuga et al., Plasmid Encoded Metallo Beta lactamase (IMP-6) Conferring Resistance to Carbapenems, Especially Meropenem. Antimicrobial Agents and Chemotherapy, p.1343-1348.

ICMR BULLETIN, Detection of Antimicrobial Resistance in common
Gram negative and Gram positive bacteria encountered in infectious diseases- An update.vol 39,No.1-3.

Jennifer, M., Andrews. 2001. Determination of minimum inhibitory concentrations. J. Antimicrobial Chemother., 48,Suppl.S15-16.

Johann, D.D., Pitout, Daniel, B., Gregson, et al. 2005. Detection of Pseudomonas aeroginosa producing Metallo Beta Lactamases in a large centralised laboratory. J. Clin. Microbio., 31293135 Vol. 43, No. 7

Lautenbach, E., Synnestvedt, M., et al., Imipenem Resistance in Pseudomonas aeroginosa: emergence, epidemiology and impact on clinical and economic outcomes. Infection control Hospital Epidemiol.

Mendiratta, D.K., Deotale, V., Narang, P.2005. Metallo beta lactamase producing Pseudomonas aeroginosa in a hospital from rural area. Indian $J$. Med. Res., 17: 14 - 7.

National Committee for Clinical Laboratory Standards. Performance standards for antimicrobials disc susceptibility tests. Approved Standard,seventh ed. NCCLS document M2-A7.Wayne, PA: National Committee for Clinical Laboratory Standards.

Navneeth, B., Sridaran, D., Sahay, D., Belwadi, M.R.S. 2002. A preliminary study on metallo beta lactamase producing Pseudomonas aeroginosa in hospitalised patients. Indian J. Med. Res., 116: $264-7$.

Nicholas Troillet, Matthew., H., Samore, and Yehuda Carmeli. 1997. Imipenem Resistant Pseudomonas aeroginosa : Risk Factors and Antibiotic susceptibility Patterns. Clin. Infectious Dis., 5: 1094-8.

Noyal, M.J.C., G.A Manezes, B.N. Harish, S. Sujatha and S.C. Parija. Simple screening tests for detection of 
carbapenemases in clinical isolates of nonfermentive Gram negative bacteria. Indian J. Med. Res., pp 707-712.

Sachie Yamoda, Toyoji okubo et al., 2003. Presence of Pseudomonas putida Strains Harbouring Plasmids Bearing the Metallo Beta Lactamase Gene bla IMP in a Hospital in Japan. J. Clin. Microbiol., p4246-4251.
Varaiya, A., Kulkarni, A., Kulkarni, M., Bhalekar, P., Dogra, J. 2008. Incidence of Metallo beta lactamase producing Pseudomonas aeruginosa in ICU patients. Indian J. Med. Res., 127(4): 398-402.

WebMD, EDTA: Uses, side effects, Interactions, Dosing.

\section{How to cite this article:}

Kanchanadevi, P., and S. Chandra Sekaran. 2016. Importance of EDTA in the Detection of Metallo Beta Lactamase from Imipenem Resistant Gram Negative Bacilli. Int.J.Curr.Microbiol.App.Sci. 5(11): 702-706. doi: http://dx.doi.org/10.20546/ijcmas.2016.511.081 